BULL. AUSTRAL. MATH. SOC.

$42 \mathrm{Al} 6,46 \mathrm{E} 99$

VOL. 27 (1983), 107-113.

\title{
FUNCTION SPACES ON THE UNIT CIRCLE
}

\author{
RICHARD J. LOY
}

In this note we give some negative results concerning the question of whether certain integrable functions on the unit circle with mean value zero are expressible as finite sums of differences $g-g_{\alpha}$ of integrable functions $g$, where $g_{\alpha}$ denotes the translate of $g$ by $\alpha$.

Let $E$ denote a Fréchet space of functions as the unit circle $\pi$. Suppose $E \subset L^{\perp}(T)$ and is translation invariant: if $f \in E$ and $\alpha \in \pi$ then $f_{\alpha}: t \mapsto f(t-\alpha)$ also lies in $E$. Let $\wedge: L^{l}(\pi) \rightarrow c_{0}(Z)$ denote the Fourier transform and suppose $\wedge$ is continuous on $E$. Set $E_{0}=\{f \in E: \hat{f}(0)=0\}$. We will always be working on $\pi$ and its dual $Z$, and so henceforth write $L^{P}$ for $L^{p}(\mathbf{T}), Z^{P}$ for $Z^{P}(Z)$ and so on. In the investigation of translation invariant linear functionals on $E$ one is led to consider the subspaces of $E_{0}$ defined by

$$
\begin{array}{ll}
\Delta_{m}(E)=\left\{f \in E_{0}: f=\sum_{i=1}^{m} g_{i}-\left(g_{i}\right)_{\alpha_{i}} \text { for some } g_{1}, \ldots, g_{m} \in E_{0},\right. \\
\Delta(E)=\bigcup_{m \geq 1} \Delta_{m}(E) .
\end{array}
$$

Indeed, a linear functional $\phi$ on $E$ is translation invariant if and only

Received 21 September 1982. 
if $\operatorname{ker} \phi \supset \Delta(E)$, and up to a scalar multiple there is only one such (namely $f \mapsto \hat{f}(0)$ ) precisely when $\Delta(E)=E_{0}$. Further, every translation invariant linear functional on $E$ is continuous if and only if $\Delta(E)$ is closed and of finite codimension in $E_{0}$.

The role of the individual $\Delta_{m}(E)$ becomes apparent from the following.

THEOREM 1. Suppose $E$ is separable and the map $E \times \pi \rightarrow E:(f, \alpha) \mapsto f_{\alpha}$ is continuous. Then $\Delta(E)$ is closed and of finite codimension in $E_{0}$ if and only if $\Delta_{m}(E)$ has finite codimension in $E_{0}$ for some $m$.

Proof. The hypotheses on $E$ ensure that each $\Delta_{m}(E)$, and $\Delta(E)$, are analytic subspaces of $E_{0}$ and so they are necessarily closed if of finite codimension. Thus codim $\Delta_{m}(E)<\infty$ for some $m$ implies $\Delta(E)$ closed and of finite codimension. Conversely, if $\operatorname{codim} \Delta(E)<\infty$ it is closed, and since $\Delta(E)=\bigcup_{m \geq 1} \Delta_{m}(E)$ some $\Delta_{k}(E)$ is nonmeagre in $\Delta(E)$. But then $\Delta(E)=\Delta_{k}(E)-\Delta_{k}(E)$ by the Pettis lemma. Since $\Delta_{2 k}(E)=\Delta_{k}(E)-\Delta_{k}(E)$ we thus have codim $\Delta_{2 k}(E)<\infty$.

Most of what is known for specific $E$ is detailed in greater generality in the survey paper [8] and a brief resumé suffices here: $\Delta_{2}\left(L^{2}\right) \varsubsetneqq \Delta_{3}\left(L^{2}\right)=L_{0}^{2}, \Delta_{2}(A)=A_{0}, \Delta\left(L^{1}\right) \neq L_{0}^{1}, \Delta_{1}\left(C^{\infty}\right)=C_{0}^{\infty}$. The $C^{\infty}$ result is proven using distributions, and consideration of the orders of the distributions involved (see [7]) enables the further conclusion $\Delta_{1}(C) \supset C_{0}^{2}$. However, the sharper result $\Delta_{1}\left(c^{E}\right) \supset c_{0}^{l+\delta}$, for any $\delta>\varepsilon>0$, is given in [2] (as is another proof of the $c^{\infty}$ result). Finally, $\Delta\left(L^{\infty}\right) \neq L_{0}^{\infty}$ is clear from the results of [10].

In [5] there is an inconclusive discussion of whether for each $f \in C_{0}$ there is some irrational $\alpha \in \mathbb{T}$ such that 
(*)

$$
\sup _{n}\left|\sum_{r=1}^{n} f(r \alpha)\right|<\infty
$$

this being equivalent to $f=g-g_{\alpha}$ for some $g \in \mathcal{C}$ by Theorem 14.11 of [3]. Here we show $\Delta_{1}(C) \neq C_{0}$ so $\left(^{*}\right)$ fails. Indeed for 'most' $f \in C_{0}$, (*) fails for 'most' $\alpha$.

THEOREM 2. (i) $C_{0} \notin \Delta_{1}\left(L^{1}\right)$ so in particuzar $c_{0} \neq \Delta_{1}(C)$.

(ii) $L_{0}^{p} \notin \Delta_{m}\left(L^{1}\right)$ if $1 \leq m<p(p-1)^{-1}$ for $1<p<\infty$.

Proof. We use the same consequence of diophantine approximation theory as has been utilized in [9], [4], [6]: if $f \in \Delta_{m}\left(L^{l}\right)$ then

$$
\lim _{k \rightarrow \infty} \inf ^{1 / m}|\hat{f}(k)|=0
$$

Thus for (i) it suffices to note that the Hardy-Littlewood function

$$
f(x)=\sum_{n=1}^{\infty} \frac{1}{n} e^{i n \log n} \cdot e^{i n x}
$$

is in $c_{0}$ yet $|\hat{f}(k)|=k^{-1}$ for $k \geq 1$.

For (ii) suppose $1 \leq m<p(p-1)^{-1}$ for some $1<p<\infty$. Then $p-2-p m^{-1}<-1$ and so $\sum n^{p-2} n^{-p / m}<\infty$, so that by [1], $\S 7.3$,

$$
f(x)=\sum_{n=1}^{\infty} n^{-1 / m} \sin n x
$$

defines $f \in L_{0}^{p}$ with $|\hat{f}(k)|=\frac{1}{2} k^{-1 / m}$ for $k \geq 1$.

We remark that $\Delta\left(L^{1}\right) \neq L_{0}^{1}$ is proved similarly to $(i i$ ) by using the function

$$
g(x)=\sum_{n=2}^{\infty} \frac{\cos n x}{\log n}
$$

Alternatively this result follows from $(i i)$ by the argument of Theorem I 
and the observation ${ }^{p} \subset L^{\perp}$ if $p \geq 1$.

Note also that if $l \leq p \leq 2$ and $f \in L^{p}$ then $f \in F_{q}$ (that is, $f \in 2^{q}$ ) for $q=p(p-1)^{-1}$ by the Hausdorff-Young inequality. Thus if $\hat{f}(0)=0$ we have $f \in \Delta_{m}\left(F_{q}\right)$ for $m>q$ by [9]. But whether or not $f \in \Delta_{m}\left(L^{p}\right)$ for $m>q$ remains open.

THEOREM 3. The set

$$
\left\{f \in C_{0}: \sup _{n}\left|\sum_{r=1}^{n} f\left(r^{2} \alpha\right)\right|=\infty \text { for } \alpha \text { in a nonmeagre set of fuzl measure }\right\}
$$

is nonmeagre in $\mathrm{c}_{0}$.

Proof. For $k=1,2, \ldots$ define

$M_{k}=\left\{f \in C_{0}: \sup _{n}\left|\sum_{\gamma=1}^{n} f(r \alpha)\right|>k\right.$ for $\alpha$ in a set of measure greater than $\left.1-k^{-1}\right\}$

Then each $M_{k}$ is open in $C_{0}$. For suppose $f \in M_{k}$, so the inequality will hold on a compact set $S$ of measure greater than $1-k^{-1}$. If $\alpha \in S$ there is $\delta(\alpha)>0$ and an integer $n(\alpha) \geq 1$ such that

$$
\left|\sum_{r=1}^{n(\alpha)} f(r \alpha)\right|>k+\delta(\alpha)
$$

Since $f$ is continuous there is thus an open neighbourhood $U(\alpha)$ of $\alpha$ such that

$$
\left|\sum_{p=1}^{n(\alpha)} f(r \beta)\right|>k+\delta(\alpha)
$$

for $\beta \in U(\alpha)$. Let $U\left(\alpha_{1}\right), \ldots, U\left(\alpha_{p}\right)$ be a finite cover of $S$ by such neighbourhoods and set $\varepsilon=\min \left\{\delta\left(\alpha_{1}\right), \ldots, \delta\left(\alpha_{p}\right)\right\}$, $m=\max \left\{n\left(\alpha_{1}\right), \ldots, n\left(\alpha_{p}\right)\right\}$. Now take $g \in C_{0}$ with $\|f-g\|<\varepsilon m^{-1}$. Then 
if $\alpha \in S$, say $\alpha \in U\left(\alpha_{j}\right)$,

$$
\begin{aligned}
\mid \sum_{r=1}^{n\left(\alpha_{j}\right)} g(r \alpha) & \geq\left|\sum_{r=1}^{n\left(\alpha_{j}\right)} f(r \alpha)\right|-\varepsilon n\left(\alpha_{j}\right) m^{-1} \\
& >k+\delta\left(\alpha_{j}\right)-\varepsilon \\
& \geq k .
\end{aligned}
$$

Thus $\left\{g \in \mathcal{C}_{0}:\|f-g\|<\varepsilon m^{-1}\right\} \subset M_{k}$.

Each $M_{k}$ is dense in $\mathrm{C}_{0}$. To see this, let $h$ denote the HardyLittlewood function defined above, normalized so $\|h\|=1$. Let $J$ denote the set of irrationals in $\pi$. Take $f \in C_{0}, \varepsilon>0$ and define the Borel set

$$
J_{1}=\left\{\alpha \in \mathrm{J} 1: \sup _{n}\left|\sum_{r=1}^{n} f(r \alpha)\right| \leq k\right\} .
$$

If $\mathbb{l}_{1}$ has measure less than $k^{-1}$ then $f \in M_{k}$. Otherwise take $0<\delta_{1}<\varepsilon$ so that by (i) of Theorem 2,

$$
\sup _{n}\left|\sum_{r=1}^{n}\left(f+\delta_{l} h\right)(r \alpha)\right|=\infty
$$

for $\alpha \in J_{1}$, and so the Borel set

$$
J_{2}=\left\{\alpha \in J I: \sup _{n}\left|\sum_{r=1}^{n}\left(f+\delta_{1} h\right)(\alpha)\right| \leq k\right\}
$$

is disjoint from $\prod_{1}$. We may continue in this manner to obtain a sequence of distinct numbers $\left\{\delta_{i}\right\}$ with $0<\delta_{i}<\varepsilon$ and disjoint Borel sets $\left\{J_{i}\right\}$. Since the $\left\{\mathrm{Jl}_{i}\right\}$ are disjoint there is some $\mathrm{Jl}_{j}$ with measure less than $k^{-1}$. But then $f+\delta_{j} h \in M_{k}$ and has distance less than $\varepsilon$ from $f$.

Let $M=\cap M_{k}$, nonmeagre in $C_{0}$ by the above. If $f \in M$ then certainly 


$$
V=\left\{\alpha \in \pi: \sup _{n}\left|\sum_{r=1}^{n} f(r \alpha)\right|=\infty\right\}
$$

has full measure. Finally, the function $\alpha \mapsto \sup _{n}\left|\sum_{r=1}^{n} f(r \alpha)\right|$ is lower semicontinuous and so is continuous at the points of a nonmeagre set $W$. It cannot be finite at any point of continuity since $V$ has full measure. Thus $W \subset V$ and the result follows.

\section{References}

[1] R.E. Edwards, Fourier series: a modern introduction, volume 1 (Holt, Rinehart and Winston, New York, 1967). See also: Second edition (Graduate Texts in Mathematics, 64. Springer-Verlag, New York, Heidelberg, Berlin, 1979).

[2] Michael Robert Hermann, "Sur la conjugaison différentiable des diffeomorphismes du cercle a des rotations", Inst. Hautes Études Sci. Publ. Math. 49 (1979).

[3] Walter Helbig Gottschalk and Gustav Arnold Hedlund, Topological dynamics (American Mathematical Society Colloquium Publications, 36. American Mathematical Society, Providence, Rhode Island, 1955).

[4] C.J. Lester, "Continuity of operators on $L_{2}(G)$ and $L_{1}(G)$ commuting with translations", J. London Math. Soc. (2) 11 (1975), 144-146.

[5] Richard J. Loy, "On the uniqueness of Riemann integration", Automatic continuity and radical Banach algebras (Lecture Notes in Mathematics. Springer-Verlag, Berlin, Heidelberg, New York, to appear) .

[6] Peter Ludvik, "Discontinuous translation invariant linear functionals on $L^{1}(G)$ ", Studia Math. 56 (1976), 21-30.

[7] Gary H. Meisters, "Periodic distributions and non-Liouville numbers", J. Fronct. Anal. 26 (1977), 68-88. 
[8] Gary Hosler Meisters, "Some problems and results on translationinvariant forms", Automatic continuity and radical Banach algebras (Lecture Notes in Mathematics. Springer-Verlag, Berlin, Heidelberg, New York, to appear).

[9] Gary H. Meisters and Wolfgang M. Schmidt, "Translation-invariant linear forms on $L^{2}(G)$ for compact abelian groups $G$ ", $J$. Funct. Anal. 11 (1972), 407-424.

[10] Joseph Max Rosenblatt, "Invariant means for the bounded measurable functions on a non-discrete locally compact group", Math. Ann. 220 (1976), 219-228.

Department of Mathematics,

Faculty of Science,

Australian National University,

PO Box 4,

Canberra, ACT 2600,

Australia. 